Articles

\title{
Empowerment of women through an innovative e-mentoring community platform: implications and lessons learned
}

\section{Antigoni Parmaxi}

\section{Christina Vasiliou}

\section{Andri Ioannou}

\section{Christiana Kouta}

Cyprus University of Technology, Cyprus

Corresponding Author.

antigoni.parmaxi@gmail.com

University of Newcastle, United Kingdom

vasi.christina@gmail.com

Cyprus University of Technology, Cyprus

andri@cyprusinteractionlab.com

Cyprus University of Technology, Cyprus

christiana.kouta@cut.ac.cy

This article presents an overview of an e-mentoring community platform that intends to promote women's empowerment. Women face the so-called glass ceiling effect, the barrier that keeps them from rising to the upper rungs of the corporate ladder, regardless of their qualifications or achievements. We aim to eliminate the stereotypical profile of women as excluded from economic, political, and professional life and promote women's empowerment, equality, and social coherence. To this aim, we aspire to develop Womenpower, an innovative e-mentoring community platform that intends to link women mentors and mentees in the areas of academia, business, and healthcare. Given the nature of this endeavor, there is a need to approach the development of the e-mentoring platform as a horizontal process and democratize the design, allowing for different perspectives of stakeholders to be heard and determine the design decisions. This article delineates the approach adopted for democratizing the design process and maximizing intended users' involvement in the development process. Finally, we conclude with implications for researchers and practitioners in Community Informatics and recommendations for promoting the participation of women in the fields of academia, business, and healthcare.

Parmaxi, A., Vasiliou, C., loannou, A., Kouta, C. (2017). Empowerment of women through an innovative e-mentoring community platform: implications and lessons learned. The Journal of Community Informatics, 13(3), 36-53.

Date submitted: 2016-05-09. Date accepted: 2017-06-04.

Copyright (C), 2017 (the author as stated). Licensed under the Creative Commons AttributionNonCommercial-ShareAlike 2.5. Available at: www.ci-journal.net/index.php/ciej/article/view/1318 


\section{Introduction}

The wage gap between men and women is gradually shrinking, yet the underrepresentation of women in higher positions still exists. Across the EU economy, women earn on average around $16.4 \%$ less than men, whereas female workers in the USA earn 77 cents for every dollar earned by male workers (Bryant, Winters, \& Matson, 2015; European Union Equality Challenge Unit, 2014; Smith, 2014). Women face the so-called glass ceiling effect "the unseen, yet unbreachable barrier that keeps minorities and women from rising to the upper rungs of the corporate ladder, regardless of their qualifications or achievements" (Federal Glass Ceiling Commission, 1995). Several researchers stress that the path for social change passes through training, education and mentoring (see for example, Amos-Wilson, 1999; Shor, 2012). To this aim, development programs and organizations such as the United Nations Development Program (UNDP) and the Association of Computing and Machinery Women (ACM-W) support mentoring and education initiatives for women, recognizing ICT as a mindchanging tool when employed and appropriately harnessed.

To take a leap towards fragmenting this glass ceiling and evade women's marginalization, we initiated the development of an e-mentoring community platform that will share good practices, successful stories and practical advice on how young women, either at university or in the workplace, can deal with these barriers. Starting from the early years of student life, we strive towards a medium for young women to direct their questions, discuss their concerns, and explore their academic and professional future through the eyes of their mentors. Our aim is to eliminate the stereotypical profile of women excluded from economic, political, and professional life (Fenton, 2000; UNESCO, 2003) and promote women's empowerment, equality, and social coherence.

Given the nature of this endeavor, there is a need to approach the development of the ementoring platform as a horizontal process and democratize the design, allowing for different perspectives of women to be heard and determine the design decisions. Bringing together women with diverse expertise in the design process encompasses both opportunities and challenges. Women's participation in the design of any ICT deployed in their community is vital to ensure its usefulness by meeting their needs, and to make sure that they enjoy the full potential of its use (Gurstein, 2003; Light et al., 2010). An essential premise of our approach is that collective communities with a diversity of expertise and different approaches to the problem will spark a powerful and dynamic interaction, promoting social creativity and building environments that move beyond traditional discipline-bound solutions. In this process, actors with different perspectives and experiences build on mutual learning to generate new understandings on how to shape a women's mentoring platform.

This study adheres to the principles of user-centered design (UCD) aspiring to contribute towards a user-friendly system that meets the needs of its intended users (Norman \& Draper, 1986). Following the participatory and cooperative design theories in Human-Computer Interaction (Muller \& Kuhn, 1993; Schuler \& Namioka, 1993) that advocate the maximum involvement of principal players, we see the active participation 
of women from the fields of academia, business and healthcare as an integral part of this project. To this aim, we found Fischer's (2001) 'Communities of interest' to be directly relevant. Communities of Interest (CoIs) encourage stakeholders with different kinds of expertise to come together for a joint venture, share diverse experiences and knowledge to the design problem and spark a powerful and dynamic interaction, promoting social creativity and innovation.

The application of CoIs adheres to the dual focus of Community Informatics (CI) as described by Stillman and Linger (2009). First, by researching the relationship between the design of a community platform and the local community, and second by implementing an ICT project in the community. In the light of these theories and the practices that they sustain, the purpose of this study is to explore the actual development of a community platform that supports e-mentoring for women. The research question that guides this study is:

- How can the design of an e-mentoring community platform for women be developed in close collaboration with its potential users and IT developers?

\section{Related Work}

\section{Gender Equality}

The under-representation of women in high-ranked positions, societal and political discussions is a pattern that occurs in several occupations across the globe. Khreisat (2009) discusses the under-representation of women in information technology and computing in the Middle East, demonstrating a steady increase in the percentage of women at all levels compared to the US. However, there is a concern related to the status of women in IT and a need for considerable effort and a combination of changes at several levels to encourage women to pursue a career in the field of computing.

Academics and researchers engage in extensive discussions regarding the need for equal opportunities in all aspects of social and political development. The legislation and policies that have been put forward have economic benefits; still, these advantages are not the sole reason for developing strategies for equal opportunities (Moreau, Osgood, \& Halsall, 2008). At the same time, research on how to narrow the gender gap is abundant. One of the ways suggested in research is through the implementation of a set of strategies for educators to close the gender gap (Scutt, Gilmartin, Sheppard, \& Brunhaver, 2013).

Hansman et al. (1999) provided further aspects to take into account when developing curriculums sensitive to gender and equality. The researchers notably suggest for girls to adopt an active expert role, including answering questions and teaching others to strengthen their identity. Tyler-Wood et al. (2012) further emphasized this argument, pointing out that exposing young students to female role models in their fields can encourage and improve their participation in related areas in the future. Providing girls 
with successful role models to look up to, can also increase their opportunities for becoming an active member of the community. Beaman et al. (2012) investigated the role model effect in women in India, suggesting that women in leadership positions may shape both students' and parents' beliefs about what women can achieve. The work of Chee, Pino, and Smith (2005) sharply describes how female students seek active participation in student clubs, pointing out that students' active involvement in such communities is positively associated with their academic performance.

Similarly, Mbarika, Payton, Kvasny and Amadi (2007) explored if ICT educational opportunities are being translated into women's empowerment and opportunities to participate in the IT work sector. Their findings demonstrated that women were optimistic and embraced ICT as a mechanism for achieving entry into a male-dominated profession such as ICT. Yet, women also desired cultural change that would make possible equal educational opportunities and ICT access and training as an imperative for their professional success. Therefore, to raise the voice of women, communicating and mentoring in the context of professional advancement seems essential (AmosWilson, 1999; Rabayah, 2010; Parcheta, Kaifi, \& Khanfar, 2013; Thomas, Bystydzienski, \& Desai, 2014).

\section{Communities of Interest}

'Communities of interest' (CoI), a notion brought forward by Fischer (2001), encapsulates the concept of bringing together stakeholders with different kinds of expertise for a joint venture. In CoIs, heterogeneous design communities work on multifaceted design problems where users act both as designers and consumers. Ultimately, CoIs contribute towards the democratization of design and innovation and foster diverse voices and active collaboration (Fischer, 2001; 2011).

One of the fundamental aspects of CoIs is the heterogeneity of participants that form the design communities. CoIs bring together representatives from different communities, with a different area of expertise in the context of a particular project, and then dissolve as the project comes to an end. These communities bring together unique experiences and perspectives about the design problem that can lead to new directions towards innovation and creativity. At the same time, this single attribute reveals a challenging path of building communication channels between the communities. Participants in CoIs share different experiences and different perspectives; thus, they need to co-create a common ground of knowledge to build on. Consequently, in the process of establishing a common ground of communication, CoIs allow for a novel understanding of the design problem and open the way for a unique perspective towards a solution.

\section{Gender Equality, Communities, and Digital Technologies}

The evolution of new information and communication technologies (ICTs) in educational and industrial settings confronts with new challenges and opportunities in supporting community building. In Islamic countries in the Middle East, digital technologies and social media allow for discussion by and social mobilization of women for equal participation in society (Shirazi, 2012). Social campaigns such as "He- 
for-She" (U.N. Women, 2014) may attract the attention of young female students but fail to sustain their interest and support them further in their professional development. However, there are natural limits generally on how much such campaigns can expand. In this context, the employment of digital technologies has the potential to support mentoring and build a community of senior and young women to exchange views and provide junior women with role behaviors. In an attempt to waive the local factor regarding practical guidance, we endeavor to develop a self-sustaining community platform to allow professional and working knowledge exchange between women mentors and mentees.

\section{Methodology}

\section{Approach}

Accessing ICT resources by itself does not ensure attainment of empowerment. There is a need to develop the appropriate approach and content that will keep women engaged in providing and receiving mentoring and ultimately improving their professional conditions. Having women actively involved in the design and implementation process of any ICT tool is crucial in optimizing its usefulness by meeting the needs of its users (Gurstein, 2003; Smith, 2015). To ensure that women's voices are heard throughout the design and development process, and the e-mentoring platform adequately addresses their needs, we formed groups of women (potential mentors and mentees) that would engage in informing the platform's design and development. With this in mind we adopted a User-Centered Design (UCD) approach that includes the following steps (see Figure 1):

a) state-of-the-art review;

b) low fidelity prototype development and evaluation;

c) focus groups with young women from academia and in the workplace;

d) a "get involved" campaign;

e) high fidelity prototype development; and

f) employment of communities of interest for evaluating the high fidelity prototype and maximizing the involvement of key stakeholders in the formulation of the platform.

From a socio-cultural perspective, the UCD process is seen as a dialogic process, with product stakeholders and end-users working together to reflect the values of the endproduct from their own perspective in a collective intellectual endeavor. This process highlights the dynamics of social practices within an interdisciplinary group, as a joint construction of knowledge (Mercer, 2004). 
Figure 1: Methodology adopted for involving critical stakeholders in the development of the Womenpower platform

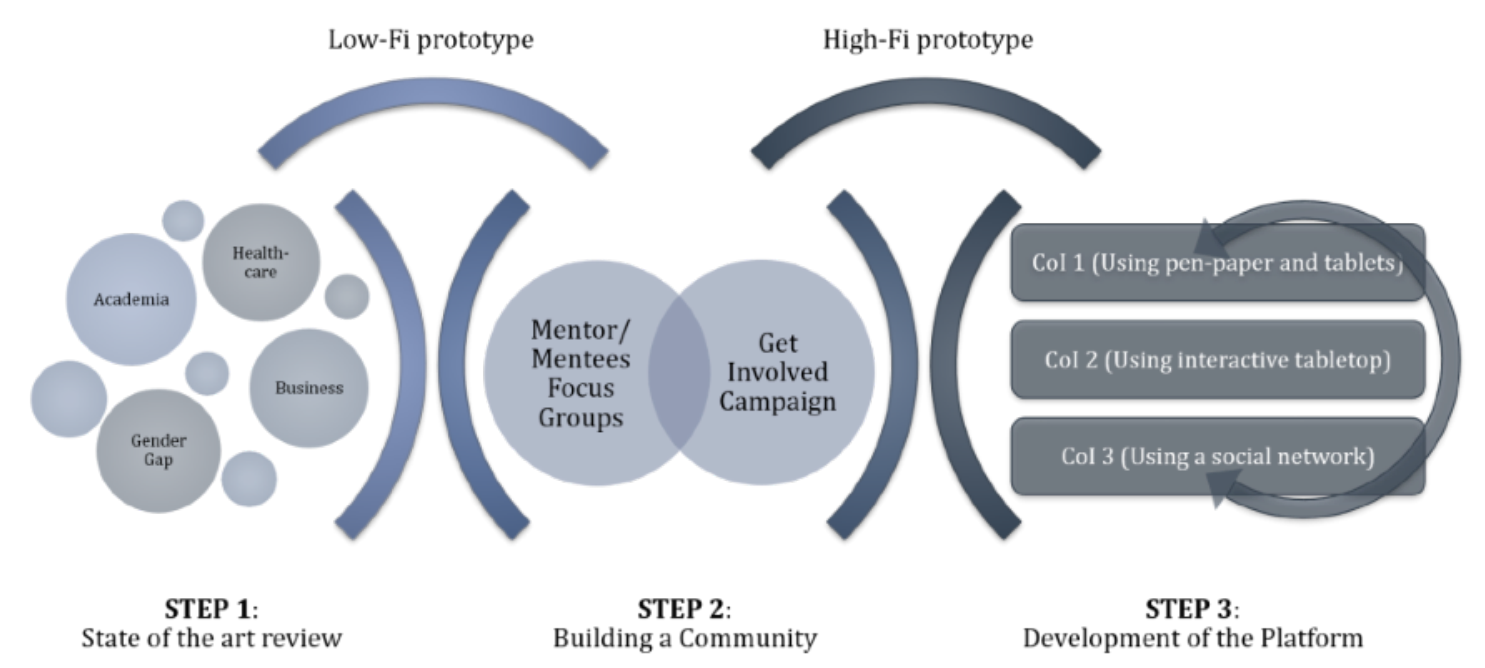

\section{Setting}

This study took place in a tertiary education institution in the republic of Cyprus. The development of the e-mentoring platform was related to an international programme funded by the UN Development Programme, Action for Cooperation and Trust in Cyprus, aimed at enhancing "Women and Dialogue".

The research team consisted of three researchers working in ICT with applications in industry and education and two researchers with backgrounds and experience in gender equality and women's health.

\section{Results}

In this section, we present the steps adopted in this project for developing an ementoring platform that intends to link women mentors and mentees in the areas of academia, business, and healthcare.

\section{State-of-the-art Review}

The first stage of our project included reviewing state-of-the-art research in the field of gender equality in academia, business, and healthcare. This phase enabled researchers to collect essential research data on the current situation of gender equality. Through this initial review, we formed the fundamental questions addressing the various aspects of gender equality in academia, business, and healthcare. Data from research informed an interview protocol, to elucidate information from women in lower and higher ranks in the academic community, healthcare and business sectors throughout three focus groups. 


\section{Low Fidelity Prototype}

In the early stages of the project, a primitive form of the platform was developed on paper. The prototype aimed to provide a first impression of the platform and inform how the platform will look and act (two snapshots are shown in Figure 2).

Figure 2: Low Fidelity Prototype snapshots of the WomenPower platform
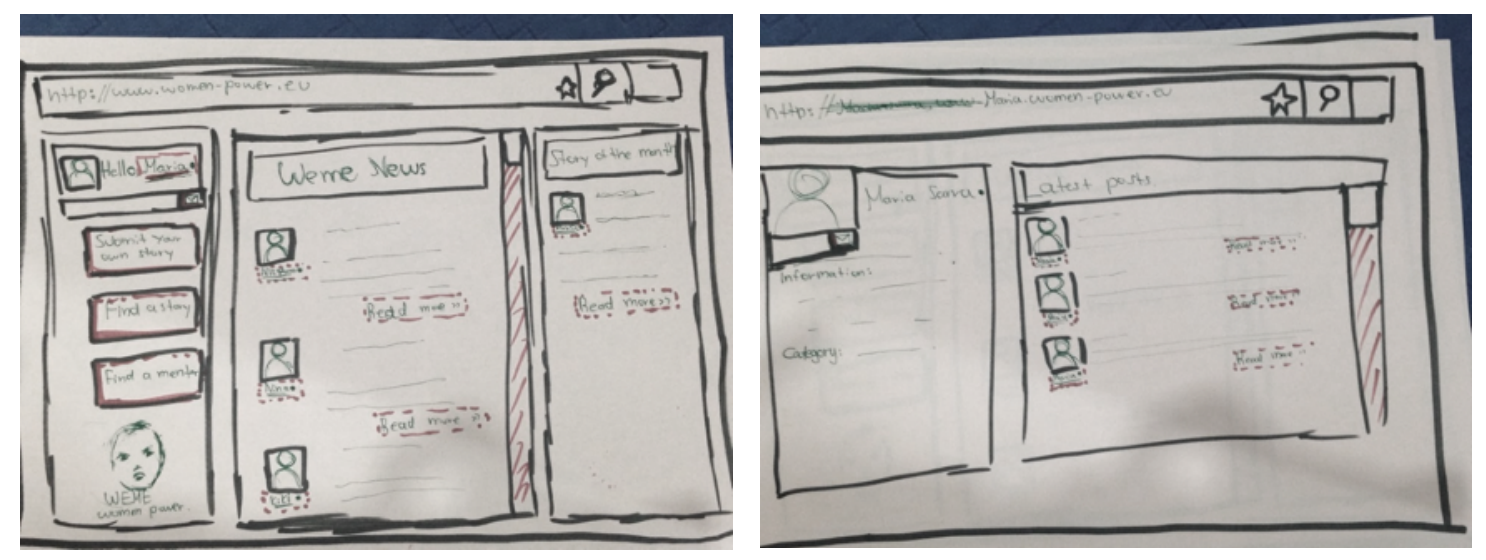

\section{Evaluation of Low Fidelity Prototype}

At the next stage, the prototype evaluation process ran on a TableTop. At first, the prototype was imported into the tabletop using the IdeaSpace application (Ioannou, Loizides, Vasiliou, Zaphiris, \& Parmaxi, 2015; Loizides, Vasiliou, Ioannou, \& Zaphiris, 2012) and facilitated simultaneous projection, discussion and commenting of the prototype. The low fidelity prototype was evaluated both by potential mentors and mentees from various areas of expertise. The low fidelity evaluation group comprised four men and two women. All four men were ICT and/or HCI experts, and their participation was considered vital to inform the design of the low fidelity prototype. One woman worked in a technology-related profession and the other in a psychology and social sciences related profession.

Throughout this phase, the potential users and experts provided feedback about the design of the platform and indicated refinements regarding idea implementation and aesthetics. The group of experts focused on the aesthetics and design of the interface and voiced the need for establishing a solid identity for this platform that would promote itself to its audience. Moreover, the group commented on the necessity for the aim and scope of the mentoring platform to be visible and understandable from the first glimpse and raise users' engagement. Strong arguments were made concerning the "sign-in" option as participants disagreed about privacy and anonymity issues.

One of the significant feedback points concerning the interface was to provide to-thepoint practical and precise information with examples of success stories. Women do not want to go through lengthy texts that take a long time to read but prefer short stories and advice that can be helpful. Further, technical experts suggested that the Graphical User 
Interface (GUI) of the platform should include more visual material and be less overloaded with textual content, to become more immersive and organized and less messy. Self-archiving of the stories and post could also be facilitated by the use of new and/or pre-defined hashtags (e.g. \#business). Users will be able to add these hashtags to their stories and posts to be able to group all posts under \#business in the same category.

Also raised were privacy and anonymity issues. Participants mentioned that the use of social networking connections (with Facebook for example) would help to reach out more people (especially the young generation); however, they pointed out that privacy issues must be seriously taken into consideration. The suggestion for each one of the users to be able to handle visibility of information was given as a means for improving the users' control in privacy settings. Experts also suggested a legal advisor should join the team to provide assistance with protection of personal data. Finally, the idea of including in the e-mentoring platform empowering quotes for women was discussed, and participants commented on their usefulness but doubted if these codes would fit in the overall mentoring platform. Participants mentioned the importance of these quotes for empowering women, yet to be meaningful, they suggested that everyone should be able to upload a quote on the website and to share it with empowering avatars with friends on a social networking site. In this way, empowering quotes can become an integral part of the e-mentoring platform for exchanging ideas and advice with other women.

\section{Focus Groups}

The focus group protocol that emerged from the state-of-the-art review conducted at the start included five thematic areas and highlighted valuable data concerning: a) gender equality at workplace; b) experiences at workplace regarding gender; c) the gender wage gap; d) the use of technology for addressing issues of inequality; and e) suggestions for the development of an e-mentoring community platform. This stage allowed us to understand the current situation in the fields of academia, business, and healthcare, the difficulties junior and senior women encounter, and how technology could help them overcome these issues (Kouta, Parmaxi \& Smoleski, 2017).

In all, three focus groups (FG) took place at a public university in the republic of Cyprus: (a) two with junior participants (FG1, $n=10$; FG2, $n=6$ ) including university students and young female professionals; and (b) one with senior participants (FG3, n= 8). In the focus groups, we invited potential mentors and mentees from the fields of business, healthcare, academia, and technology. We required the mentor women to have held a managerial, decision-making position for more than five years, and the mentees to have entered the profession in the last five years and to have had neither a managerial nor an authority position. Data collected indicated that women in the private sector encounter more problems at the workplace than women in the public sector in regards to gender equality. Young students and professionals raised the need for role models that will allow young women to overcome the standard woman profile as excluded from economic, political, and professional life. 
Participants in the mentors' focus group expressed similar views regarding gender equality, articulating equal opportunities in their workplace. On the other hand, junior students from the field of healthcare have a different view of gender equality at the workplace:

"No, we think there is gender inequality, we try, but I do not believe we can manage it." (senior-participant.4- health)

As a consequence, the treatment of employees most of the time is more questionable for women:

"Some companies have in their requirements that the woman needs to sign that [she] will not get pregnant in the next three years." (junior-participant.11health)

Moreover, different experiences were revealed between women working in public vs. the private sector. For women in the industry, gender inequality and the wage gap was more visible in their workplace.

Concerning the use of technology for developing an e-mentoring community platform, all participants were positive. Controversies arose over issues of anonymity and security. While mentors agreed that they would not like to share their names on the platform, female students stated that they would want to know who their mentors are. Moreover, mentors suggested the need to promote the platform and adopt a broad outreach strategy to be successful.

\section{Development of a high fidelity prototype}

In the next stage, the initial prototype was revised, providing a more realistic picture of the platform through the high fidelity prototype developed (see Figure 3). This phase allowed us to test the functionalities of the prototype thoroughly and proceed to the development of the platform. 
Figure 3: Snapshot of the high fidelity prototype provided to the three CoIs

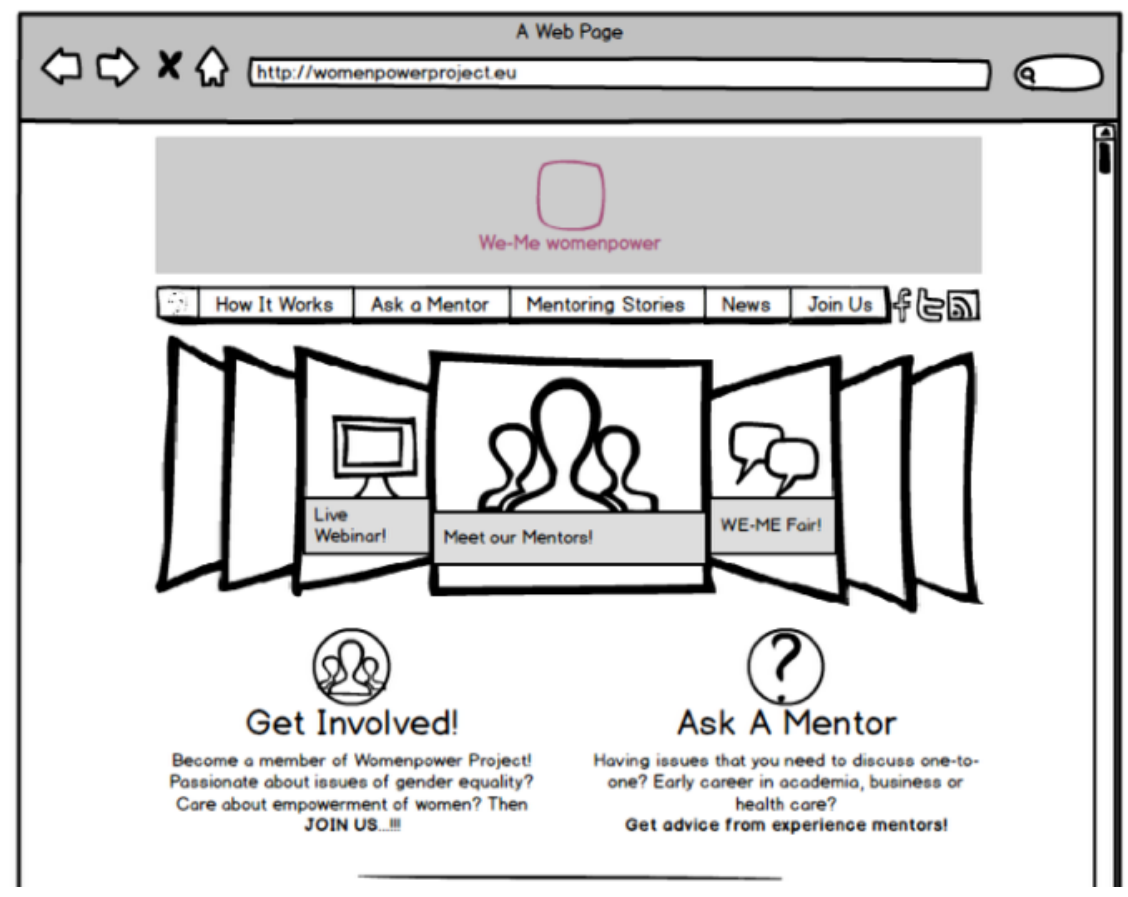

\section{Communities of Interest (CoIs)}

Following the suggestions of mentors in the focus group to incorporate a broad outreach strategy, we employed an email list of professional organizations for women in the fields of computing and machinery, business and healthcare, inviting them to express their interest in registering on the platform as mentors, mentees, or platform testers. This campaign returned more than 100 responses of women across the world who expressed interest in using and/or testing the platform.

Following this campaign, we contacted women who expressed interest to act as platform testers and invited them to join our communities of interest. As stated earlier, Communities of Interest (CoIs) bring together stakeholders with different kinds of expertise, students and women in senior positions, to come together for a joint venture. We developed three CoIs: 1) a team made up of end users that expressed their interest through the campaign. More specifically, the end users consisted of one media and communications specialist, one graphic designer, one linguistic specialist, and one software designer (5 participants from Cyprus). Computational (mobile devices) and traditional means (paper and pencil) mediated the first CoI, allowing for face-to-face interaction, thus maximizing communication between stakeholders; 2) a team made up of end-users and experts from the fields of academia, business, and technology. The second CoI was mediated by an interactive tabletop allowing for simultaneous projection, discussion and commenting on the product. The second $\mathrm{CoI}$ included four female participants from Cyprus, including one graphic designer, one web designer/ developer expert, one media and communications specialist and one software developer; and 3) a team made up of end users, gender equality and media experts, graphic, web and software designers (16 members from various countries of the world including Greece, Turkey, Malta, USA, Bulgaria, Georgia, Portugal, Italy, and United Kingdom). 
The third CoI operated within a social networking channel allowing for group members to test and discuss how to construct the community platform.

In the three CoIs, a three-stage approach was followed. First, participants watched a short video demonstrating the scope of the platform, and then were invited to work together and brainstorm on the content of the platform - using paper and pencil in CoI1; interactive tabletop in CoI2; and Google forms in $\mathrm{CoI} 3$. Then, participants were given the prototype on mobile devices (CoI1), on Tabletop (CoI2) and within the online group (CoI3) and were invited to navigate through the platform and execute a given scenario for requesting advice from a mentor on the platform (Parmaxi \& Vasiliou, 2015).

\section{Collective Brainstorming}

Results from the initial brainstorming was vivid in all three CoIs. CoI1 and CoI2 provided ideas face-to-face allowing for live discussion to grow and for ideas to be tested and negotiated. In $\mathrm{CoI} 1$ and $\mathrm{CoI} 2$ the initial brainstorming allowed group members to discuss ideas and build on the different expertise that each member held. When users were asked what they expect to see on an e-mentoring platform entitled Womenpower, they indicated that they would expect an inspirational quote. Another aspect that was considered essential in such an e-mentoring platform was the organization and structure of the content. Users noted that the content of the platform should inspire the end-user from the beginning. In a similar mindset, end users suggested linking the platform with health organizations and consultancy agents, together with FAQs, that could connect mentees to useful information.

One of the issues voiced by the end users in CoI1 was who was going to have access to this platform, how clear and simple the registration process would be and how to prevent malicious feedback or access to private data. End users raised the issue of working on the platform using nicknames, but the opinions were divergent. Building on diverse views, users decided to make anonymity optional - members chose to have their name on the platform or to remain anonymous. Mentors or Mentees should be in place to decide whether to have a public profile or appear with a nickname. Also, they noted the constant monitoring of the content in the platform and the need for members to report malicious posts and comments. In $\mathrm{CoI} 3$, participants provided their experiences concerning equality and inequality in the workplace, demonstrating conflicting ideas as some suffered from inequality in their workplace and others who did not suffer from any inequality stereotypes:

"In my current workplace, the team is made up by young and open-minded people and although the majority are males (7-4, because the initial formation of the startup company was made by a group of friends who were males), there is no hint of gender inequality in the office" (Junior participant, business, Cyprus, CoI3).

Similarly, one other participant in $\mathrm{CoI} 3$ raised the difference in the way men and women handle work: 
"For example, it is typical for men to work very long into the night and to leave family/friends only for hobby. Women work goal-oriented and not time-oriented, organize better and have more time for hobbies/family/friends. Still, this is often perceived as something negative, especially in early career. This is a large problem, which outs women under unnecessary pressure" (Senior participant, academia, Bremen, CoI3).

Women expressed their concerns about meeting their needs as professionals and mothers:

"I frequently feel that my concerns fall on deaf ears; whether this is because I am a P[project] M[manager] (and not perceived to be technical) or because I'm female, I'm not certain. I also seem to have become the de-facto "den mother" of the team. The men on the team don't seem to understand the concept of work-life balance, so I frequently find myself reminding them to do basic things like take time to sleep the next day after a late-night change. This seems ludicrous - why should I be responsible for making sure grown men take care of themselves? I certainly don't expect them to keep tabs on my welfare" (Senior participant, Ohio, Business, CoI3).

Overall, women in CoI3 were positive concerning the use of technology for addressing any inequality issues they address, yet they expressed concerns with regard to how technology will be used, together with concerns for their work.

\section{Prototype Evaluation}

Concerning the platform prototype, members provided positive feedback pointing out that it covers the aim of the platform mainly with its two functionalities on the front page. Users found the content inspiring but further indicated that the fully developed website should be in color to attract the attention of the user. One issue raised, related to the mentoring perspective of the platform, pertained to the information provided in the registration stage. The end-user should find on the page: criteria for becoming a mentor or mentee on the platform as well as a description listing the steps in the mentoring process, an inspirational message on the front page, and forum/chat functionality to facilitate communication amongst members. Moreover, designers are advised to incorporate relevant associations that members can contact, training provisions for mentors and mentees, and a suggestion box functionality that will allow participants to provide their comments to platform administrators and emphasize the unique elements of the platform - safe and professional. Overall, participants found the prototype wellconstructed, and easy to navigate and complete a given task (Parmaxi \& Vasiliou, 2015).

The continuous involvement of potential stakeholders during the design and development of the e-mentoring platform allowed for a deeper understanding of their mentoring and networking attitudes. This knowledge enabled us to construct the following two scenarios of young women using the platform for the own academic and professional development. According to Carroll and Rosson (1992), scenarios should be 
"grounded in current activities", and may include actors in a given setting, tasks to achieve a specific goal, plans, evaluation, actions, and events.

\section{Scenario A - Heather (19, Engineering Student)}

Heather, is a second-year engineering student at a private university and new member of the Womenpower platform. Heather is exploring the resources and members of the platform as she begins to search for a subject to do her major. Heather created her profile on the online platform, providing her real name and background to allow mentors to help her in this pursuit. Having set her profile, she is now browsing through the available mentors. She is seeking someone with a background in engineering that will know the available options for her current stage. She identifies two possible mentors, one from a local business and one from a university abroad, and decides to request help from both. In those emails, she shares her concerns about keeping her degree generic or following a particular direction. The local female mentor replies within a two-week period and arranges a face-to-face meeting to discuss Heather's concerns.

\section{Scenario B - Nefeli (22, Social Sciences Student)}

Raised in a traditional family, low profile. At the age of 18, she left for studies in another country and started having a different view of her role in society. She is currently in her 3rd year of Social Science studies, single and financially dependent on her family. She worked as an unpaid intern for many organizations and realized that a male with the same experience and doing the same job with her was getting paid. Even though she is extrovert, she was not courageous enough to raise the issue and discuss her problem. She searched for legislation related to gender equality in Europe, recognizing that gender equality is perceived differently in different cultures. A Google search lead her to Womenpower, a community platform linked to gender equality where she read the description and similar stories from other people all over the world. She was afraid to complain, not wanting to lose her job. Her insecurity issues were resolved when she noticed that she could register on the platform using a pseudonym. However, this did not allow the creation of her profile as there is a process of background checks to become a member of the community. She then emailed the platform administrators about her details, stating that her aim is to get informed about gender issues safely and anonymously, allowing the creation of her profile on the platform. Having set her profile, Nefeli wrote a short story describing her story and received various messages of support from women dealing with the same issue. She felt a whole community being part of her problem and having such a broad response was already comforting for her.

\section{Platform Development}

Informed by the wisdom of the CoIs, the design team made use of their comments and insights provided and incorporated them towards the development of the platform. The platform is currently active and is available here: http://womenpowerproject.eu/. 
The platform works in four simple steps as shown in Figure 4. The platform addressed the demands of its users in being colorful and providing inspirational quotes and illustrations, informative mentor profiles, one-to-one and community conversations, optional anonymity, reporting abuse and content control, a precise application form, mentoring criteria and training, and a suggestion box for encouraging further ideas for development.

Figure 4: The four-step process, demonstrating how Womenpower platform works

\section{Get Involved}
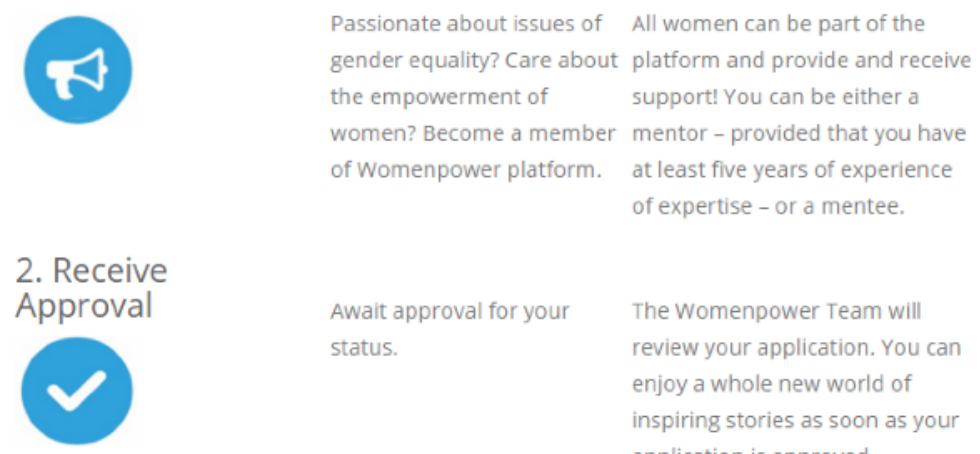

Await approval for your

\section{Ask a Mentor}
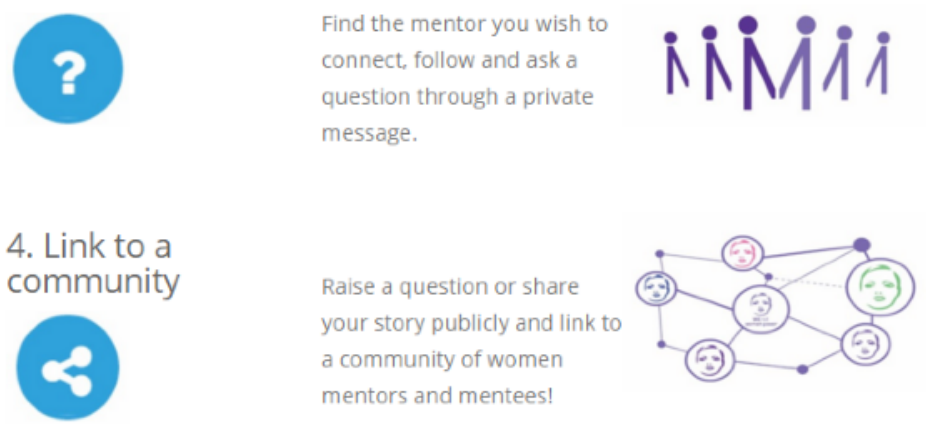

\section{Implications}

The development of any technology that aims to have an impact on the life of people in the community needs to take into consideration the different views of its potential users. In our attempt to approach the development of the e-mentoring platform as a horizontal process and democratize the design, we brought together actors with diverse expertise enriching the aspects that the design team needed to take into consideration.

Bringing together collective communities with a diversity of expertise sparked a dynamic interaction, surfacing new ideas and building an environment that moved beyond traditional discipline-bound solutions. In this process, women with different perspectives and experience, who are initially uninformed, build on mutual teaching and 
learning for generating new understandings on how to shape a women's mentoring platform.

The involvement of community stakeholders in the design and development process provided in-depth insights on how and why users may interact with the platform, identifying issues to take into consideration for its development. Diverse expertise coming from stakeholders from various fields can bring to the forefront voices from different areas and determine and resolve outstanding design aspects. End users, involved in the process from the early stages, sustained their participation informing the development of the platform. For example, during the initial stage, the feature of nicknames instead of real profile names is solving the issue of anonymity, at the same time raising more concerns in later stages regarding the falsification of personal data. In this case, the platform increased the risk of including fake profiles and misguiding the rest of the community. Platform administrators and designers should, therefore, take into consideration the differentiation between generalizing personal data (e.g. instead of the naming company, provide the topic and size of the business) and creating false personal data (e.g. entering the name of a different company).

Moreover, seeing the various perspectives from stakeholders with diverse background and expertise emerged as an essential element in the design process. In the case of the Womenpower platform, women from the area of business voiced their need to engage in both private and public discussions, while women from the field of design provided possible solutions on how the "ideal" platform would look using concrete design terminology. Hence, CoIs allowed for the abstract and robust view of the platform to come together, making space for new information and illustrations to embellish the design of the platform.

\section{Conclusion and Future Work}

This article presented how stakeholders from various fields jointly collaborated with practitioners to develop a community platform for e-mentoring, namely WomenPower. By approaching the development of such a platform as a parallel process, we allowed for the different perspectives of stakeholders to be heard in the design decision process. Currently, the platform is active (see http://womenpowerproject.eu/), and future work will indicate how different views from stakeholders were incorporated into the platform as well as how its users perceive the platform. At the moment, feedback is being received by its registered users and the platform is being revised for releasing its beta version.

CoIs can support and engage users as developers, thus bringing to the design process valuable expertise from different key stakeholders. The concept of CoIs can be incorporated in the Human-Computer Interaction and Community Informatics agenda, especially in cases that deal with building systems that will serve a variety of users from different areas of expertise. This article has also demonstrated that ICT interventions that aim to have an impact in the community need to articulate how users ascribe meaning to the issue under consideration and take stock of their views and experiences 
when designing implementations. Evaluations of such systems need to be performed embracing future users' needs from diverse cultures, areas of expertise and skills.

This work needs to be followed with an in-depth investigation of the interaction and relationships between different stakeholders within CoIs to consider how different types of technologies support different views to be heard for performing design tasks. Finally, crucial issues are demanding further attention. Key amongst these is the need to make women capable of harnessing ICT implementations efficiently and in particular for empowerment, in personal, professional and community development. Yet, it needs to be noted that being able to access ICT resources does not assure attainment of empowerment (Unwin, 2008). Women's empowerment needs to be seen using a holistic approach, framed by the appropriate political decisions that will allow promotion of women's empowerment, equality, and social coherence.

\section{Acknowledgement}

The work is funded by Mahallae, United Nations Development Programme (UNDP), Action for Cooperation and Trust (Agreement Number: 87733-MAHALLAE-01).

\section{References}

Amos-Wilson, P. (1999). The women in blue shalwar-kameez. Women in Management Review, 14(4), 128-135.

Beaman, L., Duflo, E., Pande, R., \& Topalova, P. (2012). Female leadership raises aspirations and educational attainment for girls: A policy experiment in India. Science, 335(6068), 582-586.

Bryant, M., Winters, P., \& Matson, J. (2015). Gender pay gap. Uhuru, 10(3), 10.

Carroll, J. M., \& Rosson, M. B. (1992). Getting around the task-artifact cycle: how to make claims and design by scenario. ACM Transactions on information systems, 10(2), 181-212.

Chee, K. H., Pino, N. W., \& Smith, W. L. (2005). Gender Differences in the Academic Ethic and Academic Achievement. College student journal, 39(3), 604.

European Union Equality Challenge Unit (2014). Tackling the gender pay gap in the European Union. Retrieved from http://ec.europa.eu/justice/gender-equality/files/ gender_pay_gap/gpg_brochure_2013_final_en.pdf

Federal Glass Ceiling Commission (1995). Solid Investments: Making Full Use of the Nation's Human Capital. Washington, D.C.: U.S. Department of Labor, pp. 4.

Fenton, N. (2000). The problematics of postmodernism for feminist media studies. Media, Culture and Society, 22(6), pp. 723-741.

Fischer, G. (2001). Communities of interest: Learning through the interaction of multiple knowledge systems. In Proceedings of the 24th IRIS Conference (pp. 1-14). Department of Information Science, Bergen.

Fischer, G. (2011). Social creativity: Exploiting the power of cultures of participation. In Seventh International Conference on Semantics Knowledge and Grid (SKG), 2011, pp. 1-8. IEEE. 
Gurstein, M. (2003). Effective Use: A Community Informatics Strategy Beyond the Digital Divide. First Monday, 8(12), 1 - 14.

Hansman, C. A., Jackson, M. H., Grant, D. F., \& Spencer, L. E. (1999). Assessing graduate students' sensitivity to gender, race, equality and diversity: Implications for curriculum development. College Student Journal, 33(2), 261.

Ioannou, A., Loizides, F., Vasiliou, C., Zaphiris, P., \& Parmaxi, A. (2015). Tabletop support for collaborative design: an initial evaluation of IdeaSpace. Educational Media International, 1-12.

Khreisat, L. (2009). The under-representation of women in information technology and computing in the Middle East: A perspective from Jordan. Technology in Society, 31(3), 287-294.

Kouta, C., Parmaxi, A., \& Smoleski, I. (2017). Gender Equality in Academia, Business, Technology and Health Care: A WomEnPower view in Cyprus. International Journal of Caring Sciences, 10(3), 1224.

Light, A., Ladeira, I., Roberson, J., Bidwell, N., Rangaswany, N., Sambasivan, N., \& Gitau, S. (2010). Gender matters: female perspectives in ICT4D research. Paper presented at the 4th IEEE/ACM Conference on Information and Communication Technologies and International Development (ICTD) 2010, London, UK, 13 - 16 December 2010. http://

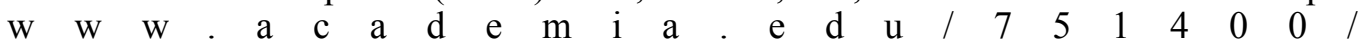
Gender_Matters_Female_Perspectives_In_ICT4D_Research

Loizides, F., Vasiliou, C., Ioannou, A., \& Zaphiris, P. (2012). Collaboratively creating a thematic repository using interactive table-top technology. In Theory and Practice of Digital Libraries (pp. 512-516). Springer Berlin Heidelberg.

Mercer, N. (2004). Sociocultural discourse analysis. Journal of applied linguistics, 1(2), $137-168$.

Moreau, M. P., Osgood, J., \& Halsall, A. (2008). Equal Opportunities Policies in English Schools: Towards greater gender equality in the teaching workforce?. Gender, Work \& Organization, 15(6), 553-578.

Mbarika, V. W., Payton, F. C., Kvasny, L., \& Amadi, A. (2007). IT education and workforce participation: A new era for women in Kenya?. The Information Society, 23(1), 1-18.

Muller, M. J., \& Kuhn, S. (1993). Participatory design. Communications of the ACM, 36(6), 24-28.

Norman, D. A., \& Draper, S. W. (1986). User Centred Systems Design. Hillsdale, NJ: Lawrence Erlbaum Associates Inc.

Parcheta, N., Kaifi, B. A., \& Khanfar, N. (2013). Gender Inequality in the Workforce: A Human Resource Management Quandary. Journal of Business Studies Quarterly, 4(3), 240-248.

Parmaxi, A., \& Vasiliou, C. (2015). Communities of interest for enhancing social creativity: The case of Womenpower platform. In Proceedings of INTED2015 conference (pp. 2838-2847).

Rabayah, K. S. (2010). Economic and social empowerment of women through ICT: a case study of Palestine. The Journal of Community Informatics, 5(3-4).

Scutt, M., Gilmartin, S., Sheppard, S., \& Brunhaver, S. (2013). Research-informed practices for inclusive science, technology, engineering, and math (STEM) classrooms: strategies for educators to close the gender gap. Am Soc Engineering Educ.

Shirazi, F. (2012). Information and communication technology and women empowerment in Iran. Telematics and Informatics, 29(1), 45-55. 
Shor, I. (2012). Empowering education: Critical teaching for social change. University of Chicago Press.

Schuler, D., \& Namioka, A. (1993). Participatory design: Principles and practices. USA: L. Erlbaum Associates Inc.

Smith, K. (2014). The Gender Pay Gap Is Just the Beginning of America's Pay Inequity Problem. Retrieved from http://www.forbes.com/sites/kylesmith/2014/04/10/thegender-pay-gap-is-just-the-beginning-of-americas-pay-inequity-problem/

Smith, R. (2015). Focus Groups as a Tool to Collect Data in a Community Informatics Project Involving Elderly Rural Women. The Journal of Community Informatics, 11(3).

Stillman, L., \& Linger, H. (2009). Community Informatics and Information Systems: can they be better connected?. The Information Society, 25(4), 255-264.

Thomas, N., Bystydzienski, J., \& Desai, A. (2014). Changing Institutional Culture through Peer Mentoring of Women STEM Faculty. Innovative Higher Education, 1-15.

Tyler-Wood, T., Ellison, A., Lim, O., \& Periathiruvadi, S. (2012). Bringing up girls in science (BUGS): The effectiveness of an afterschool environmental science program for increasing female students' interest in science careers. Journal of Science Education and Technology, 21(1), 46-55.

UNESCO (2003). UNESCO's Gender Mainstreaming Implementation Framework (GMIF) for 2002-2007, http://www.unesco.org/women

U.N. Women (2014). He For She Campaign. Retrieved from http://www.heforshe.org/

Unwin, T. (2009). Information and communication in development practices. ICT4D: Information and Communication Technology for Development, 39-75. 